\title{
A New and Reliable Guide for Studies of Neuronal Loss Based on Focal Lesions and Combinations of In Vivo and In Vitro Approaches
}

\author{
Vera Paschon ${ }^{1,29}$, Guilherme Shigueto Vilar Higa ${ }^{1,29}$, Lais Takata Walter ${ }^{1}$, Érica de Sousa ${ }^{1}$, Fausto Colla \\ Cortesão Zuzarte', Vivian Roca Schwendler Weber ${ }^{1}$, Rodrigo Ribeiro Resende ${ }^{3}$, Alexandre \\ Hiroaki Kihara ${ }^{1,2 *}$
}

1 Núcleo de Cognição e Sistemas Complexos, Centro de Matemática, Computação e Cognição, Universidade Federal do ABC, Santo André, São Paulo, Brasil, 2 Departamento de Fisiologia e Biofísica, Instituto de Ciências Biomédicas, Universidade de São Paulo, São Paulo, São Paulo, Brasil, 3 Departamento de Bioquímica e Imunologia, Instituto de Ciências Biológicas, Universidade Federal de Minas Gerais, Belo Horizonte, Minas Gerais, Brasil

\begin{abstract}
In this study, we describe a simple and reliable method to study neuroprotective effects in living and organized neural tissue. This method, which was based on retinal explants for in vivo focal lesions, was conceived as a collection of modular procedures, which can be customized for particular demands. With this model, it is possible to combine immunohistochemistry with image data analysis to track the two- or three-dimensional redistribution of proteins as a time/space function of primary cell loss. At the same time, it is possible to finely control the exposure of the tissue to specific drugs and molecules. In order to illustrate the use of the proposed method, we tested the effects of two different nanotube compounds on retinal explant viability. Transcriptome analyses can be separately performed in the lesion focus and penumbra with laser capture microdissection followed by polymerase chain reaction analyses. In addition, other common experimental drawbacks, such as high individual variance, are eliminated. With intraocular injections, treatments can be verified in vivo, with one eye serving as the experimental tissue and the other serving as the control tissue. In summary, we describe a flexible and easy method, which can be useful in combination with a broad variety of recently developed neuroprotective strategies, to study neurodegeneration.
\end{abstract}

Citation: Paschon V, Higa GSV, Walter LT, Sousa É, Zuzarte FCC, et al. (2013) A New and Reliable Guide for Studies of Neuronal Loss Based on Focal Lesions and Combinations of In Vivo and In Vitro Approaches. PLoS ONE 8(4): e60486. doi:10.1371/journal.pone.0060486

Editor: Stephen D. Ginsberg, Nathan Kline Institute and New York University School of Medicine, United States of America

Received December 17, 2012; Accepted February 26, 2013; Published April 9, 2013

Copyright: (c) 2013 Paschon et al. This is an open-access article distributed under the terms of the Creative Commons Attribution License, which permits unrestricted use, distribution, and reproduction in any medium, provided the original author and source are credited.

Funding: This work was supported by grants from Fundação de Amparo à Pesquisa do Estado de São Paulo (FAPESP), Conselho Nacional de Desenvolvimento Científico e Tecnológico (CNPq), Universidade Federal do ABC (UFABC). The funders had no role in study design, data collection and analysis, decision to publish, or preparation of the manuscript.

Competing Interests: The authors would like to disclose that corresponding author Alexandre Hiroaki Kihara is a PLOS ONE Editorial Board member. This does not alter the authors' adherence to all the PLOS ONE policies on sharing data and materials.

* E-mail: alexandrekihara@gmail.com

9 These authors contributed equally to this work.

\section{Introduction}

Molecular and cellular therapies that aim to minimize apoptotic effects reveal potential new scenarios for the treatment of neurodegenerative diseases. From nucleic acid aptamers and microRNA (miRNA) antagomirs to the specific delivery controls that are provided by carbon and peptide nanotubes, investigations that use specific concentrations and combinations of molecules and are performed in vivo are often comprehensive, particularly considering individual variability. However, neuronal and glial cell cultures offer the convenience of in vitro models, but the disruption of the original synaptic networks and the lack of an extracellular matrix environment prevent conclusions about the data from a physiological perspective [1]

When a neurodegenerative process is triggered, several mechanisms result in secondary cell death, including changes in the concentration of extracellular ions, the release of free oxygen radicals, energy depletion, high levels of the excitatory neurotransmitter glutamate, altered intracellular calcium homeostasis, and the regulation of gene expression $[2,3,4]$. Different methods have been proposed to inhibit apoptosis spread, and these could provide efficient strategies for the treatment of stroke, Alzheimer's disease, Parkinson's disease, and other neuronal diseases [5]. Monoclonal antibodies and oligonucleotide therapeutics, such as antisense and small interfering RNA, represent excellent tools for validating targets by functional inactivation of specific protein activity or by knocking out gene expression [6]. New approaches that aim to control unbalanced transcriptomes and/or proteomics, such as nucleic acid aptamers and miRNA antagomirs, have been developed $[7,8]$. In addition, the efficacy of drug delivery and its combination with nanotechnology, such as carbon and peptide nanotubes, have been extensively studied $[9,10]$.

The retina is a highly organized and easily accessible part of the central nervous system. It has a clear laminar structure and a considerable variety of cell types. Therefore, it is considered a natural brain slice, and an attractive model to study the central nervous system [11]. Moreover, the vitreous chamber acts as a capsule for drug delivery to the retina, permitting experimental 
manipulations through in vivo intraocular injections [12]. However, in vitro studies have many advantages, including the ability to highly control conditions, which allow for measurements on a cellby-cell basis, isolation from confounding systemic effects, time course flexibility, and a reduction in the number of animals required for the research. However, possible limitations include the selective loss of specific cell phenotypes/functions, changes in tissue architecture, and the questionable relevance of in vitro findings. The maintenance of preserved tissue and its original architecture and extracellular matrix provides a more realistic physiological interpretation. In this regard, the use of retinal organotypic cell cultures, which are also known as retinal explants, could be a great option because they retain many histological and biochemical features and can be maintained in vitro for several days or even weeks [1].

The retina offers an exceptional model to study trauma-induced cell death because of its easy accessibility and structural uniformity, which allows for site-restricted injuries and the reliable quantification of cellular damage [13]. As shown herein, we describe an easy and simple method that is based on mechanical trauma caused by a thin needle, resulting in precise definition of the lesion site, without global traumatization observed in ischemia models [14,15]. Moreover, whole retinas may be dissected after different time points, enabling reliable quantitative comparisons of miRNA, gene expression, and protein concentrations. When combined with other techniques, such as lactate dehydrogenase $(\mathrm{LDH})$ assays [16], laser capture microdissection (LCM) [17,18], or terminal deoxynucleotidyl transferase (TdT)-mediated 2-deoxyuridine-5-triphosphate (dUTP) nick-end labeling (TUNEL) [19], this method greatly reduces the individual variance and can be customized for particular demands. In addition to analyses of the explant medium, several methodologies can be applied to the retinal explant itself, including histological examinations after specific treatments. In Fig. 1, we present an overall view of the possibilities that can be performed using the described method.
Although some of the procedures presented herein have been previously described, the combination of these techniques with mechanical lesions of the retina is described for the first time in this study as a general guide to evaluate neuronal loss.

\section{Methods}

\section{Ethics Statement}

The experiments were conducted in accordance with guidelines of the National Institutes of Health and the Brazilian Scientific Society for Laboratory Animals. The experimental protocol was approved by the Ethics Committee in Animal Experimentation of the Institute of Biomedical Sciences/University of São Paulo (ICB/USP).

\section{Animal Procedures}

In this study, we utilized male chicks (Gallus gallus) that were 715 days of age and rats (Rattus novergicus) that were 50-60 days of age. All animals were bred and housed in a vivarium with free access to food and water and were kept on a 12:12-h light/dark cycle with lights on at 06:00 a.m. Chick colonies were maintained at $32-35^{\circ} \mathrm{C}$ in the first week and $29-32^{\circ} \mathrm{C}$ and $26-29^{\circ} \mathrm{C}$ in the second and third weeks, respectively. However, rats were maintained constantly at $22-24^{\circ} \mathrm{C}$. Chicks were fed CN Frango Inicial (20\% protein, CN Rações, Bom Jardim, RJ, Brazil), and rats were fed Nuvilab CR-1 (23\% protein, Quimtia Brasil, Colombo, PR, Brazil) Animals were not handled before the lesion procedure, except for cage cleaning.

Animals were anesthetized with ketamine $(10 \mathrm{mg} / 100 \mathrm{~g}$ of body weight, i.p., Parke-Davis, Ann Arbor, MI, USA) and xylazine (1 mg/100 g of body weight, i.p., Miles Inc., West Haven, CT, USA). In order to confirm that the animals were anesthetized, a paw was pressed to test the pain withdrawal reflex. Thereafter, animals were subjected to 6 local mechanical lesions in the retina. The lesions were made with a thin needle (28-gauge, $12.7 \mathrm{~mm}$, BD Medical-Diabetes Care, Franklin Lakes, NJ, USA), which

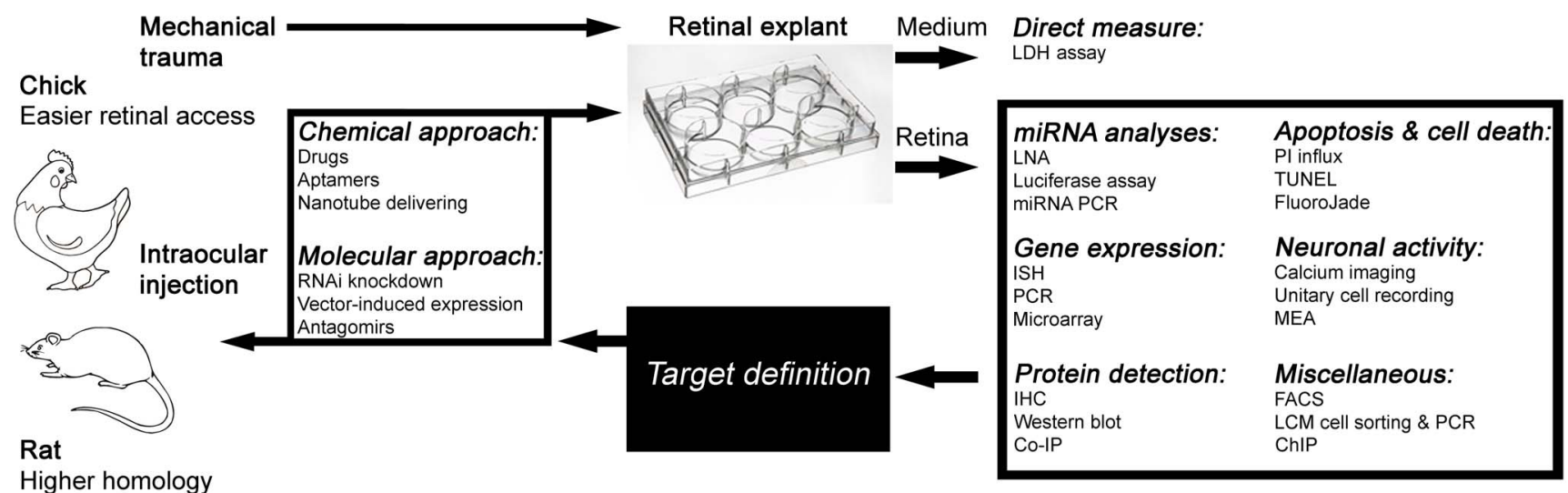

Higher homology

with human

Figure 1. Summary of the proposed guide for neurodegeneration studies with a retinal trauma model. Retinal lesions can be produced with a thin needle in different species, including chicks and rats. Chick retina offers easy access because of its size, even in embryonic ages, whereas experiments that are conducted in rats may be of clinical relevance due to their higher homology with humans. After different post-lesion time points, the animals were euthanized, and the retinas were utilized in different methods. Retinal explants can be treated with different classes of molecules, including aptamers, antagomirs, or carbon nanotubes. The lactate dehydrogenase (LDH) concentration in the culture medium can be used as a reliable probe of cell viability. Afterwards, the retinas can be utilized in a wide range of experimental analyses, from epigenetics, such as microRNA (miRNA) activity to chromatin alterations, and live cell activity recording, which employs calcium imaging or multielectrode arrays. Once the role of a particular target molecule or gene is determined during apoptosis progression, in vivo validation can be performed by comparing control vs. experimental conditions in the same animal. The use of both eyes from the same animal allows for simple statistical analyses such as paired $t$-tests.

doi:10.1371/journal.pone.0060486.g001 
crossed the cornea, lens, vitreous, and retina. The lesions were performed without completely removing the needle from the eye in order to cause minimal damage to the cornea. After different periods, animals were euthanized with an overdose of ketamine (30 mg/100 g, i.p.) and xylazine (2 mg/100 g, i.p.). The eyecups were removed, and the retinas were gently dissected for different methodologies.

\section{Immunohistochemistry}

Seven-day-lesioned chick eyes were fixed for $30 \mathrm{~min}$ in $4 \%$ paraformaldehyde (PFA, Sigma-Aldrich Co. LLC, St. Louis, MO, USA) diluted at $<60^{\circ} \mathrm{C}$ in phosphate-buffered saline (PBS; $0.1 \mathrm{M}$, $\mathrm{pH} 7.3$ ) and cryoprotected in $30 \%$ sucrose solution for $24 \mathrm{~h}$ at $4^{\circ} \mathrm{C}$. After embedding the eyes in Optimum Cutting Temperature compound (OCT, Sakura Finetek USA, Inc., Torrance, CA, USA), they were cut transversally $(12 \mu \mathrm{m})$ on a cryostat, and 1 in 5 sections were sampled from the nasal to the temporal direction. For the analysis, we examined 3-5 sections in each animal $(n=3)$. Retinal sections were blocked for $30 \mathrm{~min}$ in a solution containing $10 \%$ normal goat serum, $1 \%$ bovine serum albumin, and $0.3 \%$ Triton-X 100 in PBS.

To characterize the reactive gliosis in the retinal lesion, a mouse monoclonal antibody that was raised against glial fibrillary acidic protein (GFAP) was employed (\#G3893, 1:4,000, Sigma-Aldrich Co. LLC) in order to identify Müller glial cells, as described in other studies [20]. Controls for the experiments consisted of the omission of primary antibodies; no staining was observed in these cases. After several washes, retinal sections were incubated with goat antiserum against mouse IgG that was tagged to Alexa TM 488 (Life Technologies Corporation, Grand Island, NY, USA 1:250-1:500) and diluted in PBS containing 0.3\% Triton X-100 for $2 \mathrm{~h}$ at room temperature. Retinas were incubated with propidium iodide $(1: 4,000)$ for $10 \mathrm{~min}$ and washed thrice in PBS for $5 \mathrm{~min}$. Finally, sections were coverslipped with Vectashield Mounting Media (Vector Laboratories, Inc., Burlingame, CA, USA).

\section{Image Analysis}

Slides were analyzed with a Nikon TS100F inverted microscope, and photomicrographs were acquired with NIS-elements AR 3.2 64-bit software (Nikon Instruments Inc., Melville, NY, USA). Image analysis was performed with Image-Pro Plus software (Media Cybernetics, Inc., Bethesda, MD, USA), as previously described [21]. In summary, after channel separation (RGB) of the color images, we performed two different analyses: (i) counting cells/nuclei: after proper setting of the size and brightness, the software identified discrete elements and automatically counted labeling that was displayed in the image, and thus, artifacts and background labeling could be identified and discarded and (ii) bitmap analysis: $x-y$ axis analyses generated numerical-appended data files corresponding to the pixel values. The bitmap analysis was used to view the pixel values of the active window (or area of interest) in a numerical format, in which the values corresponded to the brightness of the pixels. This matrix was exported to Excel (Microsoft, Redmond, WA, USA) for appropriate mathematical computations. The numerical data generated a histogram, which essentially averaged the labeling intensities at different retinal locations. Photomicrographs and charts were prepared with Adobe Photoshop CS2 (Adobe Systems Inc., San Jose, CA, USA).

\section{Carbon Nanotube Synthesis}

Single-wall carbon nanotubes (SWCNTs) were prepared by the arc discharge method with a $\mathrm{Co} / \mathrm{Ni}(0.6 / 0.6 \mathrm{~atm})$ catalyst with helium at a total pressure of 500 Torr, with the arc generated by a current of 200A/20V [22,23]. After synthesis, an indispensable asgrown SWCNT purification (approximately 95\%) process was performed. One gram of SWCNTs was refluxed with $3 \mathrm{M} \mathrm{HNO} 3$ at $120^{\circ} \mathrm{C}$ for $32 \mathrm{~h}$, centrifuged at 7,000 rpm, and washed with distilled water in order to purify the SWGNTs. Nitric acid oxidation of the carbon nanotubes exerted a dual role: this treatment was performed in order to decorate the SWCNT surface with - COOH groups and to result in short SWCNTs (length, 50$500 \mathrm{~nm}$ ). The final solution was dried for $12 \mathrm{~h}$ in an oven at $60^{\circ} \mathrm{C}$. Finally, $0.75 \mathrm{~g}$ of highly pure $\mathrm{COOH}-\mathrm{SWCNT}$ s was obtained. Subsequently, SWCNTs were fluorinated to a $\mathrm{C}: \mathrm{F}$ ratio of approximately $2.4: 1$ by direct fluorination at $150^{\circ} \mathrm{C}$ according to a previously reported procedure [24].http://pubs.rsc.org/en/ content/articlehtml/2005/cc/b509257d - cit14.

The fluoronanotubes were functionalized with a reaction with the appropriate amine in the presence of a base catalyst. Fluoronanotubes (approximately $15 \mathrm{mg}$ ) were sonicated in dimethylformamide $(30 \mathrm{~mL})$ for $10 \mathrm{~min}$, resulting in complete dispersion and the formation of a dark solution. A solution of 2aminoethanethiol hydrochloride $(200 \mathrm{mg})$ in dimethylformamide $(20 \mathrm{~mL})$ and $4-5$ drops of pyridine (catalyst) was then added. The reaction mixture was stirred (under $\mathrm{N}_{2}$ ) for $3 \mathrm{~h}$ at $90^{\circ} \mathrm{C}$. The reaction mixture was filtered through a $0.2-\mu \mathrm{m}$ Cole Parmer Teflon membrane and washed with acetone to ensure complete removal of unreacted 2-aminoethanethiol, reaction by-products, and solvent. The thiol-SWCNTs (SWCNT-SH) were dried overnight in a vacuum at $70^{\circ} \mathrm{C}$.

\section{Retinal Explants and LDH Assays}

Whole retinas were carefully dissected and gently placed in culture dishes containing explant medium. This medium was prepared with $1 \times$ Basal Medium Eagle, $5 \%$ bovine fetal serum, and $1 \%$ glutamine. Retinal damage was assessed by measuring LDH that was released in the explant medium, which was expressed as a percentage of total $\mathrm{LDH}$ released, as reported previously [25]. $\mathrm{LDH}$ is an enzyme that catalyzes the conversion of pyruvate to lactate with concomitant interconversion of nicotinamide adenine dinucleotide hydrogen and nicotinamide adenine dinucleotide. This methodology is usually associated with studies that are conducted on both cardiac and striatal striated muscle. In the nervous tissue, the quantification of $\mathrm{LDH}$ that was released into the culture medium was used as an indicator of cell viability [26].

Chick retinal explants $(n=6)$ were conditioned in the culture medium for $4 \mathrm{~h}$ at room temperature. After 1, 2, or $4 \mathrm{~h}, 400 \mu \mathrm{L}$ of the culture medium was collected and centrifuged at $100 \times g$ for 5 min. LDH was measured in aliquots of supernatant with colorimetric quantification with a specific biomedical kit (Labtest Diagnostica SA, Lagoa Santa, MG, Brazil). The results were obtained by measuring light absorbance at $500 \mathrm{~nm}$ with a spectrophotometer.

\section{TUNEL Assays}

One-day-lesioned chick eyes were fixed for $30 \mathrm{~min}$ in $4 \%$ PFA in PBS (0.1 M; pH, 7.3) and cryoprotected in 30\% sucrose solution for $24 \mathrm{~h}$ at $4^{\circ} \mathrm{C}$. After embedding in OCT compound, they were cut transversally $(12 \mu \mathrm{m})$ on a cryostat, and 1 in 5 sections were sampled from the nasal to the temporal direction, as previously described $[27,28]$. For the analysis, we examined 3-5 sections in each animal $(n=3)$ at the lesioned sites or at the corresponding locations in the control eyes. Labeling was accomplished by incubation in TdT buffer for $10 \mathrm{~min}$ at room temperature, followed by incubation with biotinylated dUTP 


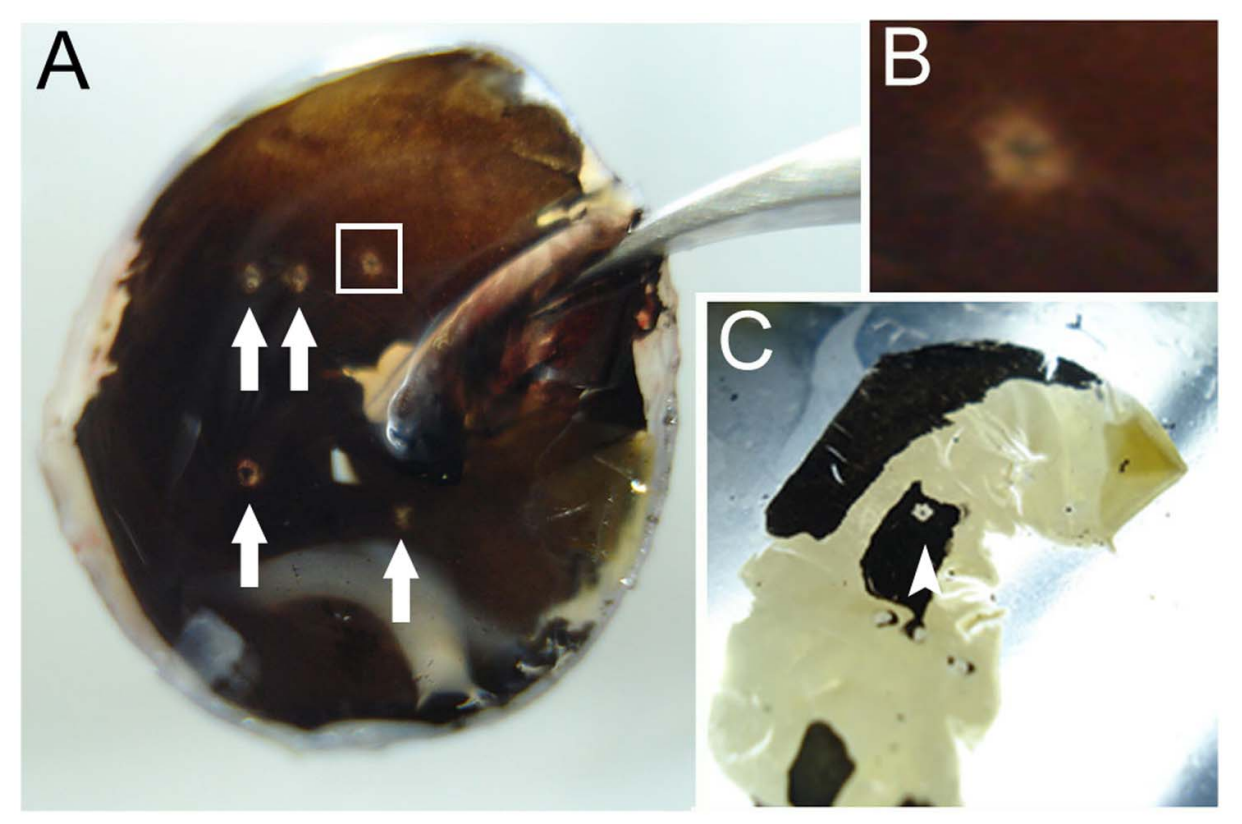

Figure 2. Identification of focal lesions in the chick retina. Mechanical lesions were produced with a thin insulin needle that crossed the cornea, lens, vitreous, retina, and epithelium. (A) After removing the vitreous, it was possible to identify the focal lesions in the middle retina (arrows), close to the pecten oculi. (B) High magnification of the selected area in the white square in A is shown. Although there is a semitransparent aspect to the retina, the lesion borders are well defined because the pigmented epithelium was also perforated. (C) Even after the complete removal of the retina from the sclera, it was possible to visualize the lesions containing some surrounding epithelium (arrowhead). Complete removal of the pigmented epithelium is facilitated by incubating the retina in cold phosphate-buffered saline. doi:10.1371/journal.pone.0060486.g002

(Roche Molecular Biochemicals, Mannheim, Germany). The reaction was terminated in stop-reaction buffer $(10 \%, 0.1 \mathrm{M}$ ethylenediaminetetraacetic acid, $\mathrm{pH}$, in water) and washed in PBS. Counterstaining of the retinas was performed with $4^{\prime}, 6-$ diamidino-2-phenylindole (DAPI) by incubating the sections at room temperature for $10 \mathrm{~min}$. The sections were mounted with a ProLong Antifade kit (Life Technologies Corporation).

\section{LCM Cell Asorting and Polymerase Chain Reaction (PCR) Analyses}

LCM of stained cryostat sections has been used to separate distinct cell populations to determine specific gene expression patterns in tissues with a complex mixture of cell types [29]. However, RNA degradation is a major drawback in most common fixation protocols recommended for tissue processing prior to LCM [30]. Therefore, specific fixation and RNA isolation protocols have been developed for LCM [17,31]. We demonstrated the use of this technology with chick retinas, which were dissected $(\mathrm{n}=3)$, fixed in 2\% PFA for $15 \mathrm{~min}$, and then incubated with $30 \%$ sucrose for $1 \mathrm{~h}$. Cryostat sections (5-10 per retina; 10$12 \mu \mathrm{m}$ thick) were obtained in the nasal to temporal direction and rapidly stained with cresyl violet.

To examine gene expression in specific cell populations, we performed LCM (LaserScissors 390/20, Cell Robotics International, Inc., Albuquerque, NM, USA) in chick retinal sections. After selection of the region with the laser, different procedures can be employed to extract the tissue from the microscope slide. Herein, to isolate the microdissected cells, the laser was focused on the microscope slide. A single shot at maximum laser power provides sufficient energy to completely detach the isolated fragment, which is collected by electrostatic attraction by a thin plastic coverslip that is placed on top of the specimen without direct contact. With this procedure, fragments of the inner nuclear layer were microdissected from transverse retinal slices $(\mathrm{n}=3)$, and attempts were made to avoid contamination from other layers $[29,32]$. RNA was isolated with TRIzol $^{\circledR}$ according to the instructions provided by the manufacturer (Life Technologies Corporation) and the specific changes described in previous studies [17]. Total RNA was treated with 20 units of DNase I GE Healthcare Biosciences, Piscataway, NJ, USA) to eliminate residual DNA. mRNA was reverse transcribed with the SuperScript II (Life Technologies Corporation) standard protocol. Endpoint PCR was conducted with primers for chick glyceraldehyde 3-phosphate dehydrogenase (GAPDH; forward: 5'-GGAGCGTGACCCGAGCAACA-3'; reverse: 5'-ACAGCTTCCGAGAGGGGCGA-3').

\section{Statistical Analysis}

For the LDH experiments employing SWCNTs, we used twoway analysis of variance (ANOVA) with the factors of time and treatment, which was followed by pair-wise comparisons with Tukey's HSD test. When we compared LDH release from control vs. lesioned retinas and the results of TUNEL analyses, we used paired $t$-tests.

\section{Results}

\section{Examining the Injured Retina}

Due to its size, eyecups were easily removed from the chick head. When we employed rats, the eyes were cut off from the head with small tweezers. After detaching the vitreous, visualization of the focal lesions in the retina was facilitated with magnifying glasses. As shown in Fig. 2, it was possible to observe the focal lesions right after the dissection procedure. Removal of pigmented epithelium was accelerated by incubating the retina in cold PBS. The lesions were well defined and surrounded by epithelial 
infiltration. Even after complete dissection of the retina from the eye, focal lesions were observed in the middle of the retina, which contained some epithelium (Fig. 2).

When the retina is processed for quantitative methods, such as real-time PCR or western blotting, the pigmented epithelium should be completely removed.

\section{Reactive Gliosis within the Focal Lesion}

Differences in immunolabeling can be detected in microscope sections, but variations in the immunolabeling can be misleading. Therefore, the presence of affected and nonaffected regions within the same section is a reliable way to compare specific changes in protein distribution. We performed immunofluorescence assays to evaluate reactive gliosis in the focus of the lesion and the gradient in the surrounding areas (Fig. 3) and we demonstrated that changes in protein distribution that are caused by the neurodegenerative process can be assessed with the proposed model. Therefore, GFAP distribution was analyzed in vertical sections of 7-day-lesioned chick retinas. Chick retinal slices were counterstained with propidium iodide to facilitate the visualization of the nuclear layers [33]. As shown in Fig. 3, this model allows for the spatial definition of the lesion with high reproducibility, permitting visualization of the focus, the penumbra, and the adjacent areas. As expected, within the lesion focus, we observed intense GFAP labeling due to reactive gliosis $(\mathrm{n}=3,3-5$ samples per retina). With software that is dedicated to image analysis, it is possible to determine the optical density within the focus, penumbra, and adjacent areas (Fig. 3).

\section{LDH Release is a Reliable Probe of Retinal Cell Viability}

As an example of LDH assay employment in neurodegeneration studies, we tested the effects of carbon nanotubes on concentration of LDH released by undamaged rat retinas. LDH is released in explant medium over time, allowing for the measurement of its concentrations at different time points. As expected, we observed increasing absorbance values after 1, 2, and $4 \mathrm{~h}$ (Fig. 4). However, we did not detect significant differences in concentrations of $\mathrm{LDH}$ released from explants incubated with two distinct nanotube compounds $(10 \mu \mathrm{g} / \mathrm{mL})$. As a positive control in the experiment, we compared concentrations of LDH released from 1-day-lesioned vs. nonlesioned rat retinal explants. Lesioned retinas released more $\mathrm{LDH}$ after $4 \mathrm{~h}$ in culture $(\mathrm{n}=6, P<0.01)$. To test detection linearity, 4-h explant medium was diluted to different concentrations and subjected to an $\mathrm{LDH}$ assay. Linear regression analyses of plots generated from medium serial dilutions ranging from $100 \%$ to $25 \%$ revealed a high correlation $\left(\mathrm{R}^{2}=0.9786\right)$, indicating detection linearity (Fig. 4). Taken together, these results indicated that $\mathrm{LDH}$ is a reliable probe for retinal cell viability in vitro.

\section{LCM Cell Sorting in the Retina Followed by PCR Analysis}

We prepared samples for LCM to select fragments from a specific retinal layer. Microdissected cells from the inner nuclear layer of the chick retina were isolated and carefully harvested to perform RNA isolation, cDNA synthesis, and PCR analysis. We were able to detect GAPDH bands at the expected molecular weight, confirming the mRNA integrity (Fig. 5). The importance of this result is two-fold. First, these results showed that the retrieval of mRNA could be achieved in the fixed retina, as described in previous studies. Moreover, these results disclosed that it is possible to assess changes in gene expression during neurodegeneration in specific neuronal cell types.
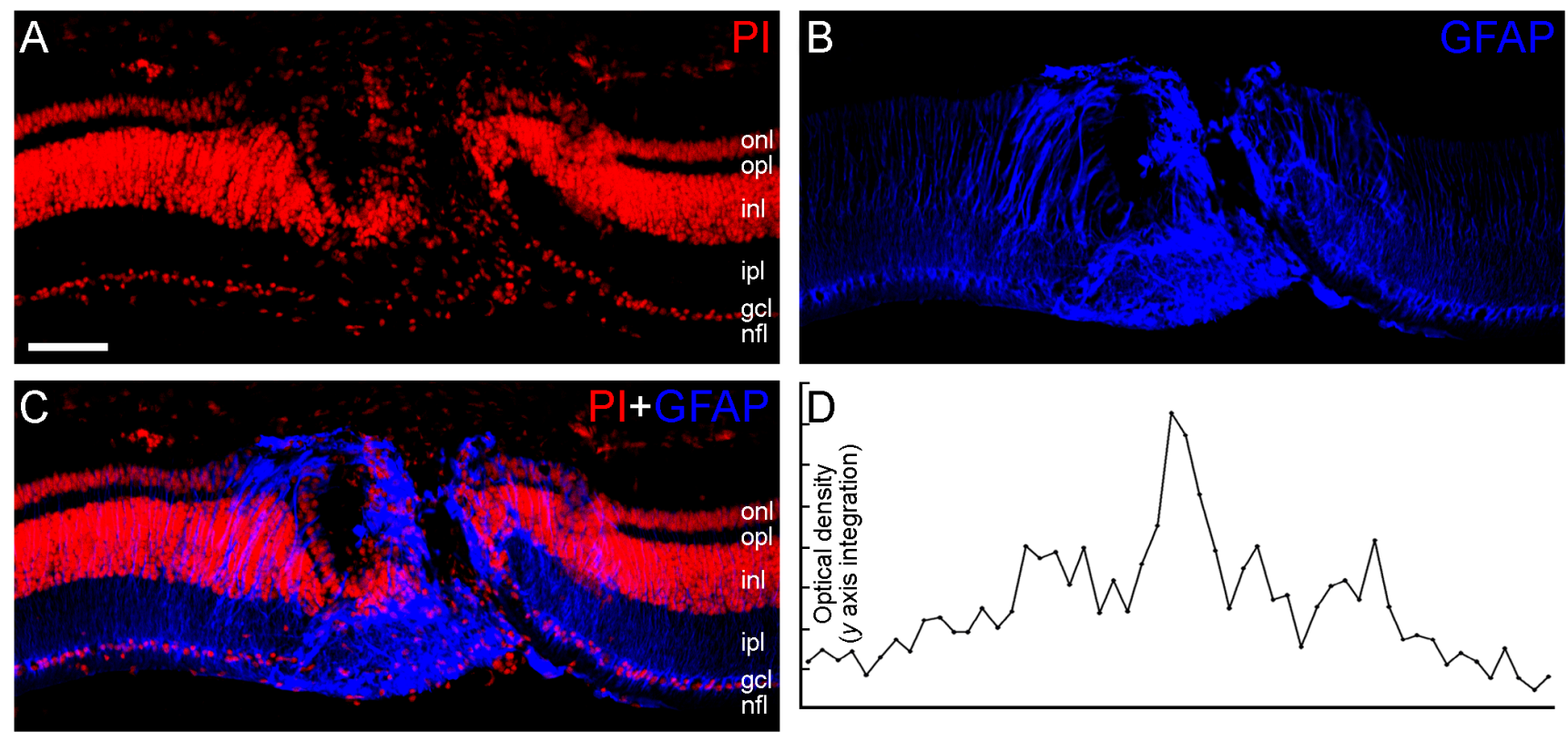

Figure 3. Glial fibrillary acidic protein (GFAP; blue) immunolabeling in vertical sections of 7-day-lesioned chick retinas counterstained with propidium iodide (red). (A-C) Vertical sections of chick retina were counterstained with propidium iodide (red) to facilitate visualization of the nuclear layers. In order to validate the focal lesion model, GFAP (blue) immunolabeling was conducted. We detected typical intense GFAP labeling, representing the reactive gliosis process, which decreased toward the penumbra and adjacent areas $(n=3,3-5$ samples per retina). (D) With the use of a pixel analysis, quantification of the optical density along the focus, penumbra, and adjacent areas was possible. The labels indicate the approximate location of the outer nuclear layer (onl), the outer plexiform layer (opl), the inner nuclear layer (inl), the inner plexiform layer (ipl), the ganglion cell layer ( $\mathrm{gcl})$, and the nerve fiber layer (nfl). Scale bar: $60 \mu \mathrm{m} . \mathrm{G}$.

doi:10.1371/journal.pone.0060486.g003 
A

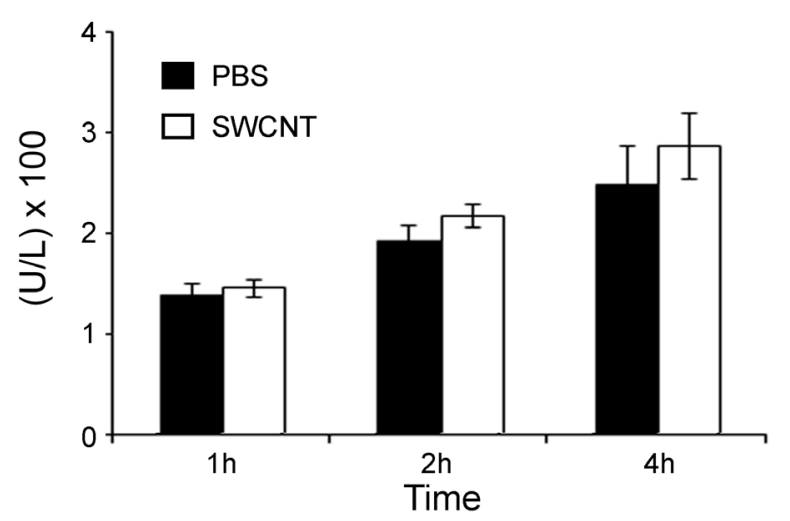

C

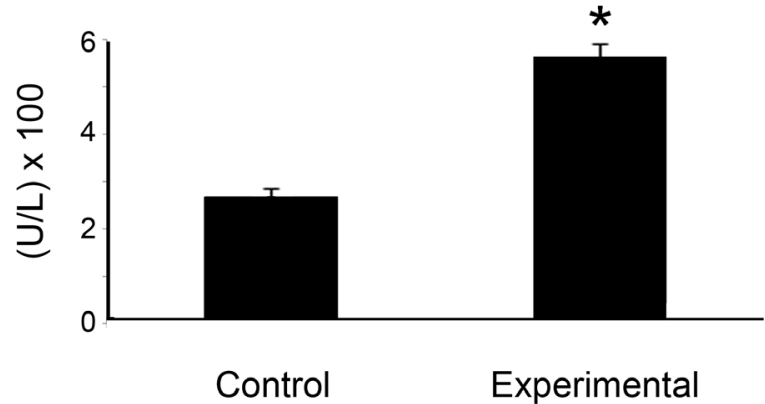

$\mathrm{B}$

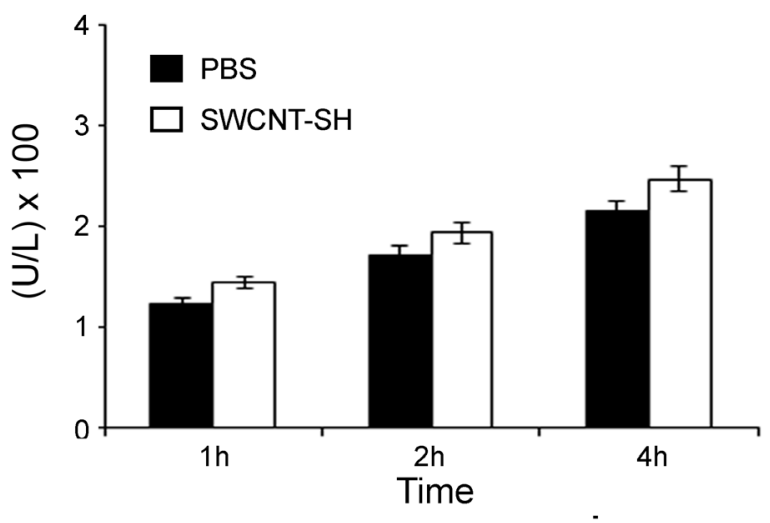

$\mathrm{D}$

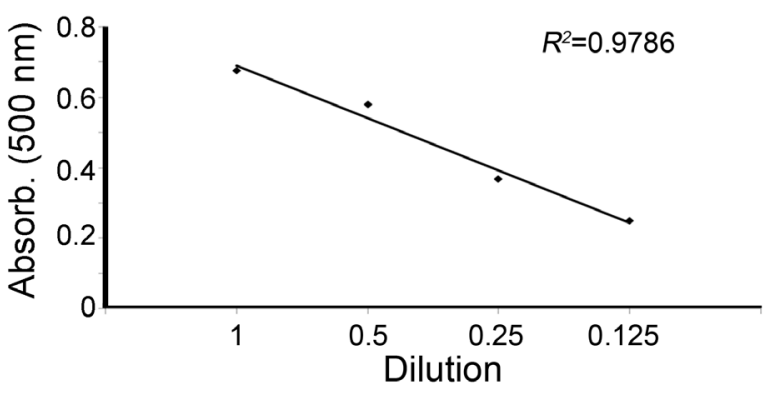

Figure 4. Quantification of cell viability with a lactate dehydrogenase (LDH) assay in rat retinal explants. We determined the effects of two distinct carbon nanotube compounds on retinal explants, (A) single-wall carbon nanotubes (SWCNT) and (B) thiol-single-wall carbon nanotubes (SWCNT-SH). LDH accumulated in the explant medium over time. Thus, we observed crescent values of absorbance $(500 \mathrm{~nm})$ after 1,2 , and $4 \mathrm{~h}$. We did not detect significant differences in LDH concentrations that were released by undamaged retinas incubated with the two distinct nanotube compounds (10 $\mu \mathrm{g} / \mathrm{mL})$. (C) As a positive control, we compared the concentrations of LDH that was released from 1-day-lesioned vs. nonlesioned retinal explants. As expected, lesioned retinas released more $\mathrm{LDH}\left(\mathrm{n}=6,{ }^{*} P<0.01\right)$. (D) Explant medium was diluted in different concentrations and tested in an LDH assay. A linear regression analysis of the plots generated from medium serial dilutions ranging from $100 \%$ to $25 \%$ revealed high correlations between the concentrations and the readouts $\left(R^{2}=0.9786\right)$, indicating detection linearity.

doi:10.1371/journal.pone.0060486.g004

A

Tissue preparation

\begin{tabular}{l|l|l}
\multicolumn{1}{c|}{ Option 1: } & \multicolumn{1}{c}{ Option 2: } & \multicolumn{1}{c}{ Option 3: } \\
\hline i) Fresh retina & i) Fresh retina & i) Fresh retina \\
ii) $2 \%$ PFA $(15 \mathrm{~min})$ & ii) Frozen in & ii) $95 \%$ Ethanol or \\
iii) $30 \%$ Sucrose $(1 \mathrm{~h})$ & liquid nitrogen & $3 \%$ PFA (overnight) \\
iv) Sectioning & iii) Sectioning & iii) $70 \%$ Ethanol (24 h) \\
v) Cresyl violet & iv) $70-95 \%$ Ethanol & iv) Paraffin embedding \\
or H\&E staining & or 100\% Acetone & v) Sectioning \\
& (1 min) & vi) Cresyl violet \\
& v) Cresyl violet & or H\&E staining \\
& or H\&E staining &
\end{tabular}
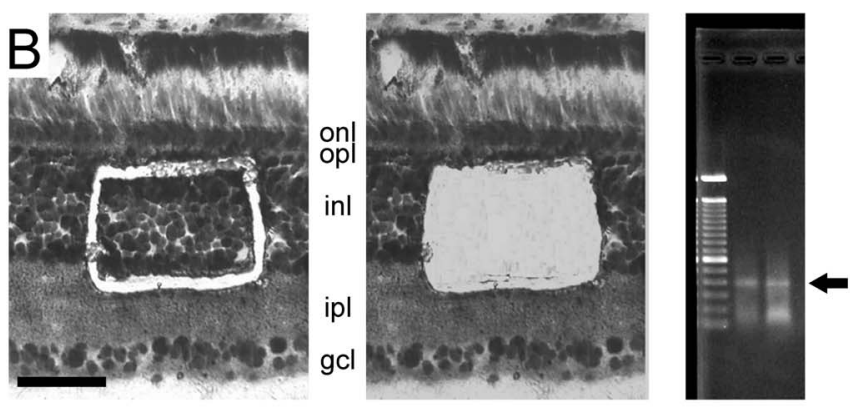

laser selection of area of interest

Figure 5. Laser capture microdissection (LCM) followed by reverse transcription-polymerase chain reaction (RT-PCR) allows for quantitative gene expression analyses of specific cell populations. (A) Different protocols were described to prepare samples for LCM cell sorting, including (i) fixation procedures for sectioning with a cryostat, (ii) sectioning that is followed by fixation in specimen, and (iii) preparation for paraffin embedding. Microdissection of the inner nuclear layer in 10-12 $\mu \mathrm{M}$ chick retina cryostat slices exemplifies the use of this technique. (B) The retina was rapidly counterstained with cresyl violet to permit the identification and isolation of a specific retinal layer. In order to isolate the microdissected cells, the laser was focused on the microscope slide. A single shot at maximum laser power provides sufficient energy to completely detach the isolated fragment, which is collected by electrostatic attraction to a thin plastic coverslip that is placed on top of the specimen. RNA isolation was performed with protocols that were modified from conventional commercial kits. After 35 cycles of conventional PCR, we detected specific bands for chick glyceraldehyde 3-phosphate dehydrogenase (GAPDH; arrow). In addition to fresh tissue, this technique can be performed in retinal explants. Scale bar: $60 \mu \mathrm{m}$. Partial reproduction from our previous work [44].

doi:10.1371/journal.pone.0060486.g005 


\section{Evaluation of Apoptotic Dynamics}

As we have shown, an LDH assay may provide a quantitative measure of cell viability, which reflects the integrity of neuronal membranes. However, the apoptotic process is classically related to fragmentation of the cell nucleus, and detection of this particular condition is frequently performed with a TUNEL assay [27]. Although TUNEL has been shown to be a reliable method for the detection of apoptotic cells, analyses of TUNEL labeling do not have suggested standards. Thus, fine and precise evaluations of prospective neuroprotective effects could be quite difficult. To overcome this hindrance, we developed a simple and accurate method to model apoptosis spread (Fig. 6).

Herein, as an example, we evaluated the effects of the gap junction blocker carbenoxolone $(\mathrm{CBX})$ on the distribution of apoptotic cells in chick retinal explants. One-day-lesioned retinas were maintained in culture for $4 \mathrm{~h}$ at room temperature. By taking the lesion focus as a reference, the distribution of apoptotic nuclei was typically narrower in retinas that were treated with CBX
$(100 \mu \mathrm{M})$, although some variations in spatial distribution were observed. To test the consistency of this result, we employed two different quantification methods. First, we plotted the number of apoptotic nuclei that were counted at different distances from the lesion focus. Next, data plots were submitted to a linear regression with the least squares approach, which generated parameters, such as $R^{2}$ and $R$, and a first-order equation $(\mathrm{y}=\mathrm{ax}+\mathrm{b})$ for each experimental condition. As a second approach, the values from bitmap analyses were submitted to the same procedure. The bitmap analysis was used to view the pixel values of active windows in numeric format, in which the values corresponded to the brightness of the pixels. The mean of the angular coefficient from the first-order equation $(a)$ was calculated for each experimental condition $(\mathrm{n}=3)$ as a parameter of apoptotic spread (Fig. 6).

For the number of apoptotic cells, the absolute value of $a$ was higher with $\mathrm{CBX}$ treatment $(-1.83 \pm 0.35, \mathrm{P}<0.05)$ compared with PBS treatment $(-0.40 \pm 0.06)$. With similar procedures, we were able to detect significant changes in the $a$ values that were
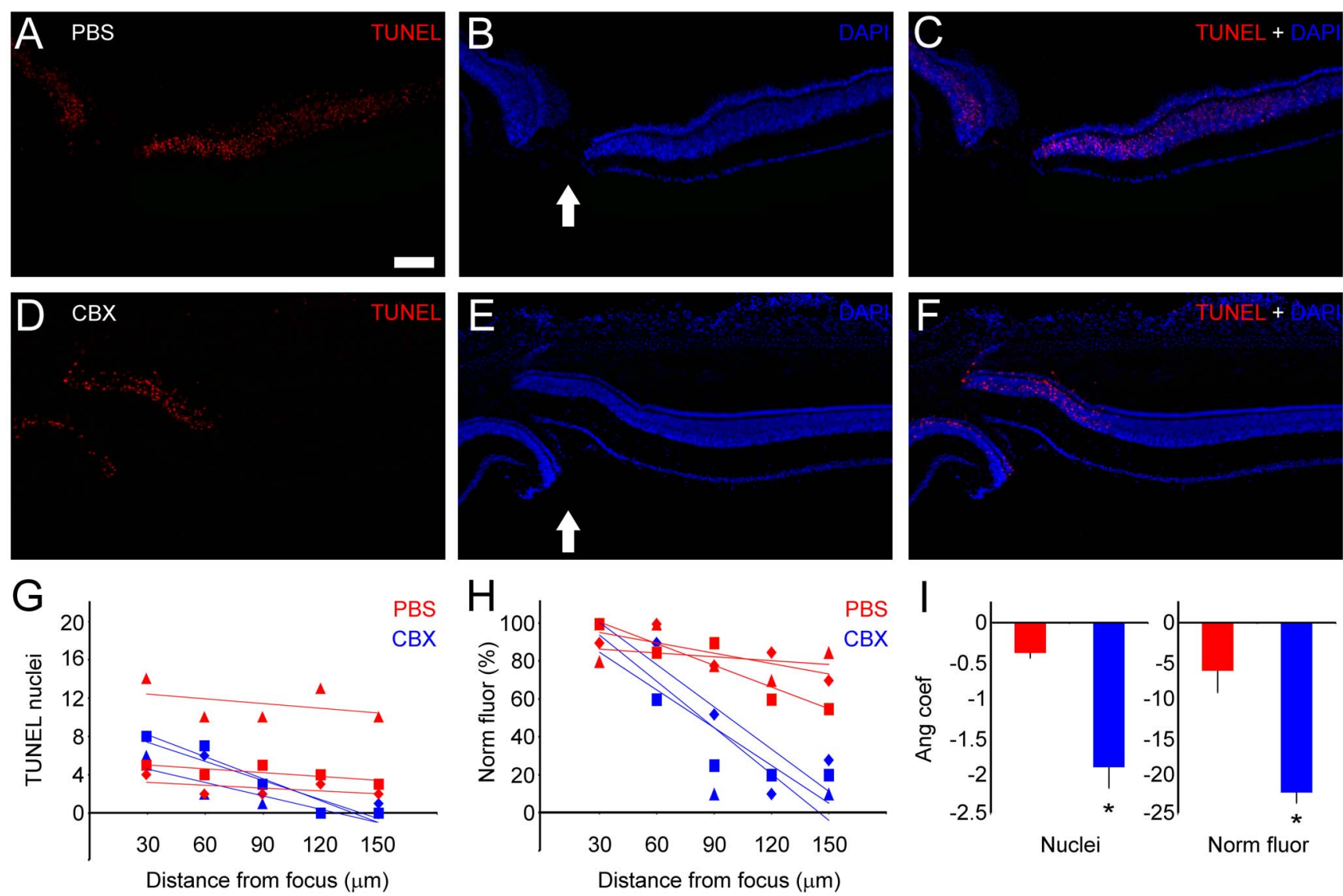

Figure 6. Modeling apoptotic spread in degenerating tissue. Explants of 1-day-lesioned chick retinas were maintained for $4 \mathrm{~h}$ in culture media with $(A-C)$ phosphate-buffered saline (PBS) or (D-F) $100 \mu \mathrm{M}$ of carbenoxolone (CBX). Afterwards, transverse sections were submitted to terminal deoxynucleotidyl transferase (TdT)-mediated 2-deoxy-uridine-5-triphosphate (dUTP) nick-end labeling (TUNEL) in order to characterize the apoptotic spatial pattern. In each image, it was possible to localize the focus of the lesion (arrows). (G) In order to determine whether gap junction blockers caused changes in the distribution of apoptotic cells, we counted the number of TUNEL-positive nuclei that were located as far as $150 \mu \mathrm{m}$ away from the focus of the lesion. The values were plotted according to the distance from the focus, and they were subjected to linear regression with the least squares approach, generating mathematical parameters, such as $R^{2}$ and $R$, and the first-order equation $(y=a x+b)$. $(H)$ The same procedure was undertaken with values from the pixel analysis. An $x-y$ axis bitmap analysis was used to view the pixel values in numeric format, in which the values corresponded to the brightness of the pixels. Considering the distribution of TUNEL-positive nuclei, the means of the angular coefficient from the first-order equation $(a)$ were calculated for each experimental condition $(n=3)$. (I) Compared with the control $(-0.40 \pm 0.06)$, the absolute value of $a$ was higher with $C B X$ treatment $(-1.83 \pm 0.35, P<0.05)$. With regard to the values from the bitmap pixel analysis, we detected significant changes in the $a$ angular coefficient when comparing PBS $(-6.33 \pm 2.77)$ vs. CBX $(-22.3 \pm 1.3, \mathrm{P}<0.05)$ treatments. ${ }^{*} P<0.05$ in a paired $t$ test. Scale bar: $60 \mu \mathrm{m}$.

doi:10.1371/journal.pone.0060486.g006 
obtained from the bitmap pixel analysis comparing PBS $(-6.33 \pm 2.77)$ vs. $\mathrm{CBX}(-22.3 \pm 1.3, \mathrm{P}<0.05)$ treatments (Fig. 5). Taken together, these results revealed that apoptotic spread could be compared and quantified with a combination of in vivo lesions, in vitro treatments, and mathematical analyses. The effects of CBX on apoptotic spread and the presence of gap junction proteins in dying neurons have been previously reported by our group [28].

\section{Discussion}

Immunohistochemistry has been a valuable tool in both the diagnosis and research of infectious and degenerative diseases [34,35]. Differences in immunolabeling can be detected in microscope sections, but these variations can be misleading. In this regard, the presence of experimental and control regions within the same section is a reliable way to compare specific changes in protein distribution in a degenerative tissue. As we demonstrated, the proposed model of retinal degeneration allowed for the visualization of the focus, the penumbra, and the adjacent areas within the same image. As an example, we were able to detect undisputed changes in GFAP labeling, which characterize the process of reactive gliosis. Indeed, gliosis is a hallmark of neurodegenerative conditions in several regions, including the retina [36]. GFAP immunolabeling spanned all the retinal layers within the lesion focus and decreased toward the penumbra areas. In adjacent areas, GFAP labeling was similar to that seen in nonlesioned retinas. Müller glial cells undergoing reactive gliosis are believed to alter the local environment of neurons and provide additional structural integrity to the retina at the site of injury by inducing changes in intermediate filament constituents [37].

In addition to gliosis, another common feature in the neurodegenerative process is that secondary cell death is frequently more detrimental than the loss caused by the first injury. Pharmacological agents have been tested in an attempt to decrease the apoptotic program and/or spread, despite the fact that the balance of the benefits and detriments of these strategies remains under debate [38,39]. In this regard, in vitro approaches are quite useful for testing the efficacy of specific molecules. Indeed, neuronal, glial cell, and mixed cultures offer the convenience of in vitro models, but the disruption of the original organization and the lack of the extracellular matrix prevent a more realistic and pathophysiological interpretation of the data. With the use of retinal explants, cells retain many histological and biochemical features and can be maintained for several days or even weeks $[1,40]$. It has been reported that long-lasting neuronal cell cultures remain viable for months, thus adding a few easy procedures to traditional protocols [41]. However, although the time in culture cannot be compared, explants have an important advantage: they are relatively easy to establish because the tissue is directly transferred to a culture plate containing explant medium following the dissection. However, shorter periods in culture have additional advantages. An optic nerve axotomy does not severely affect ganglion cell survival in the time scale that we evaluated in culture [42]. Similar effects were observed in other regions of the nervous system [43], indicating that neurons remained viable for shorter periods after axotomy.

As we demonstrated, an $\mathrm{LDH}$ assay can be performed with the explant medium after different time points in culture. The measures of the LDH amounts allow for an evaluation of cell membrane stability, which in turn reflects cell viability. As expected, we observed that LDH release was higher in 1-daylesioned retinas compared with that in the respective controls. In fact, previous retinal studies have reported that secondary cell loss occurs predominantly by apoptosis and mostly within 24 and $36 \mathrm{~h}$ [13]. LDH assays are highly compatible with other contemporary approaches that are based on RNAi, antagomirs, aptamers, and overexpression systems and aim to examine neuroprotection. Furthermore, retinal explants could be useful for testing newly developed techniques of drug delivery, such as those that are combined with peptide or carbon nanotubes $[9,10]$.

After the role of a particular molecule in cell death/survivor is determined, in vivo testing can be performed with intraocular injections. In this case, presumably neuroprotective effects can be verified after acquiring data provided by in vitro observations. Again, this has several advantages, such as easy access to the retina, a well-known morphology, and the fact that both experimental and control conditions can be tested in the same animal.

In summary, the combination of focal lesions in the retina and an in vitro approach permits the use of several distinct and contemporary techniques, which can be quite useful in studies examining neuroprotection.

\section{Acknowledgments}

The authors would like to thank Caroline Margonato, Otávio Casado, Marjorie dos Santos, Cássia Ortolani, for scientific discussions, Caroline Gonçalves, Michael Araújo and Gisele da Silva for technical assistance.

\section{Author Contributions}

Conceived and designed the experiments: VP GSH RRR AHK. Performed the experiments: VP GSH LTW ÉS FCZ VRW. Analyzed the data: VP GSH RRR AHK. Contributed reagents/materials/analysis tools: AHK. Wrote the paper: VP GSH RRR AHK.

\section{References}

1. Seigel GM (1999) The golden age of retinal cell culture. Mol Vis 5: 4.

2. Lynch DR, Dawson TM (1994) Secondary mechanisms in neuronal trauma. Curr Opin Neurol 7: 510-516.

3. Kermer P, Klocker N, Bahr M (1999) Neuronal death after brain injury. Models, mechanisms, and therapeutic strategies in vivo. Cell Tissue Res 298: 383-395.

4. Saba R, Goodman CD, Huzarewich RL, Robertson C, Booth SA (2008) A miRNA signature of prion induced neurodegeneration. PLoS One 3: e3652.

5. Sureda FX, Junyent F, Verdaguer E, Auladell C, Pelegri C, et al. (2011) Antiapoptotic drugs: a therapautic strategy for the prevention of neurodegenerative diseases. Curr Pharm Des 17: 230-245.

6. Eckstein F (2007) The versatility of oligonucleotides as potential therapeutics. Expert Opin Biol Ther 7: 1021-1034.

7. Selvamani A, Sathyan P, Miranda RC, Sohrabji F (2012) An antagomir to microRNA Let7f promotes neuroprotection in an ischemic stroke model. PLoS One 7: e32662.

8. Nastasijevic B, Wright BR, Smestad J, Warrington AE, Rodriguez M, et al. (2012) Remyelination induced by a DNA aptamer in a mouse model of multiple sclerosis. PLoS One 7: e39595.

9. Lee HJ, Park J, Yoon OJ, Kim HW, Lee do Y, et al. (2011) Amine-modified single-walled carbon nanotubes protect neurons from injury in a rat stroke model. Nat Nanotechnol 6: 121-125.

10. Silva GA (2006) Neuroscience nanotechnology: progress, opportunities and challenges. Nat Rev Neurosci 7: 65-74.

11. Becker D, Bonness V, Mobbs P (1998) Cell coupling in the retina: patterns and purpose. Cell Biol Int 22: 781-792.

12. Magharious MM, D’Onofrio PM, Koeberle PD (2011) Optic nerve transection: a model of adult neuron apoptosis in the central nervous system. J Vis Exp.

13. Striedinger K, Petrasch-Parwez E, Zoidl G, Napirei M, Meier C, et al. (2005) Loss of connexin36 increases retinal cell vulnerability to secondary cell loss. Eur J Neurosci 22: 605-616.

14. Rawanduzy A, Hansen A, Hansen TW, Nedergaard M (1997) Effective reduction of infarct volume by gap junction blockade in a rodent model of stroke. Neurosurg Focus 2: E1; discussion 1 p following E1.

15. Oguro K, Jover T, Tanaka H, Lin Y, Kojima T, et al. (2001) Global ischemiainduced increases in the gap junctional proteins connexin 32 (Cx32) and Cx36 in hippocampus and enhanced vulnerability of Cx32 knock-out mice. J Neurosci 21: 7534-7542. 
16. Wang L, Liu L, Shi Y, Cao H, Chaturvedi R, et al. (2012) Berberine induces caspase-independent cell death in colon tumor cells through activation of apoptosis-inducing factor. PLoS One 7: e36418.

17. Kihara AH, Moriscot AS, Ferreira PJ, Hamassaki DE (2005) Protecting RNA in fixed tissue: an alternative method for LCM users. J Neurosci Methods 148: 103-107.

18. Marselli L, Thorne J, Dahiya S, Sgroi DC, Sharma A, et al. (2010) Gene expression profiles of Beta-cell enriched tissue obtained by laser capture microdissection from subjects with type 2 diabetes. PLoS One 5: e11499.

19. Wines-Samuelson M, Schulte EC, Smith MJ, Aoki C, Liu X, et al. (2010) Characterization of age-dependent and progressive cortical neuronal degeneration in presenilin conditional mutant mice. PLoS One 5: e10195.

20. Torelli S, Sogos V, Marzilli MA, D’Atri M, Gremo F (1989) Developmental expression of intermediate filament proteins in the chick embryo retina: in vivo and in vitro comparison. Exp Biol 48: 187-196.

21. Kihara AH, Santos TO, Paschon V, Matos RJ, Britto LR (2008) Lack of photoreceptor signaling alters the expression of specific synaptic proteins in the retina. Neuroscience 151: 995-1005.

22. Ladeira MS, Andrade VA, Gomes ER, Aguiar CJ, Moraes ER, et al. (2010) Highly efficient siRNA delivery system into human and murine cells using singlewall carbon nanotubes. Nanotechnology 21: 385101.

23. Silva EE, Colleta HHM, Ferlauto AS, Moreira RL, Resende RR, et al. (2009) Nanostructured 3-D collagen/nanotube biocomposites for future bone regeneration scaffolds. Nano Research 2: 462-473.

24. Khabashesku VN, Billups WE, Margrave JL (2002) Fluorination of single-wall carbon nanotubes and subsequent derivatization reactions. Acc Chem Res 35: 1087-1095.

25. Romano C, Price MT, Olney JW (1995) Delayed excitotoxic neurodegeneration induced by excitatory amino acid agonists in isolated retina. J Neurochem 65 : 59-67.

26. Keilhoff G, Wolf G (1993) Comparison of double fluorescence staining and LDH-test for monitoring cell viability in vitro. Neuroreport 5: 129-132.

27. Belmonte MA, Santos MF, Kihara AH, Yan CY, Hamassaki DE (2006) LightInduced photoreceptor degeneration in the mouse involves activation of the small GTPase Racl. Invest Ophthalmol Vis Sci 47: 1193-1200.

28. Paschon V, Higa GS, Resende RR, Britto LR, Kihara AH (2012) Blocking of Connexin-Mediated Communication Promotes Neuroprotection during Acute Degeneration Induced by Mechanical Trauma. PLoS One 7: e45449.

29. Emmert-Buck MR, Bonner RF, Smith PD, Chuaqui RF, Zhuang Z, et al. (1996) Laser capture microdissection. Science 274: 998-1001.
30. Qin Y, Heine VM, Karst H, Lucassen PJ, Joels M (2003) Gene expression patterns in rat dentate granule cells: comparison between fresh and fixed tissue. J Neurosci Methods 131: 205-211.

31. Foss RD, Guha-Thakurta N, Conran RM, Gutman P (1994) Effects of fixative and fixation time on the extraction and polymerase chain reaction amplification of RNA from paraffin-embedded tissue. Comparison of two housekeeping gene mRNA controls. Diagn Mol Pathol 3: 148-155.

32. Luo L, Salunga RC, Guo H, Bittner A, Joy KC, et al. (1999) Gene expression profiles of laser-captured adjacent neuronal subtypes. Nat Med 5: 117-122.

33. Kihara AH, Santos TO, Osuna-Melo EJ, Paschon V, Vidal KS, et al. (2010) Connexin-mediated communication controls cell proliferation and is essential in retinal histogenesis. Int J Dev Neurosci 28: 39-52.

34. Ramos-Vara JA (2005) Technical aspects of immunohistochemistry. Vet Pathol 42: 405-426.

35. Friedrich S, Schwabe K, Klein R, Krusche CA, Krauss JK, et al. (2011) Comparative morphological and immunohistochemical study of human meningioma after intracranial transplantation into nude mice. J Neurosci Methods 205: 1-9.

36. Ganesh BS, Chintala SK (2011) Inhibition of reactive gliosis attenuates excitotoxicity-mediated death of retinal ganglion cells. PLoS One 6: e18305.

37. Dyer MA, Cepko CL (2000) p57(Kip2) regulates progenitor cell proliferation and amacrine interneuron development in the mouse retina. Development 127 : 3593-3605.

38. Borgens RB, Liu-Snyder P (2012) Understanding secondary injury. Q Rev Biol 87: 89-127.

39. Buss RR, Sun W, Oppenheim RW (2006) Adaptive roles of programmed cell death during nervous system development. Annu Rev Neurosci 29: 1-35.

40. Zhang SS, Fu XY, Barnstable CJ (2002) Tissue culture studies of retinal development. Methods 28: 439-447.

41. Potter SM, DeMarse TB (2001) A new approach to neural cell culture for longterm studies. J Neurosci Methods 110: 17-24.

42. Bull ND, Johnson TV, Welsapar G, DeKorver NW, Tomarev SI, et al. (2011) Use of an adult rat retinal explant model for screening of potential retinal ganglion cell neuroprotective therapies. Invest Ophthalmol Vis Sci 52: 3309 3320

43. Akahori Y, Horie H (1997) IGF-I enhances neurite regeneration but is not required for its survival in adult DRG explant. Neuroreport 8: 2265-2269.

44. Bittencourt JC, Elias CF (2007) Métodos en Neurociência. São Paulo: Roca. 